\title{
Measuring micronutrient intakes at different levels of sugar consumption in a population in transition: the Transition and Health during Urbanisation in South Africa (THUSA) study
}

\author{
MacIntyre UE, PhD, Professor of Nutrition \\ Department of Human Nutrition, University of Pretoria, Pretoria \\ Venter CS, DSc, Professor of Nutrition \\ North-West University, Centre of Excellence for Nutrition, Potchefstroom \\ Kruger A, PhD, Professor of Nutrition \\ North-West University, Africa Unit for Transdisciplinary Health Research, Potchefstroom \\ Serfontein M, MSc Dietetics, MSc Student \\ North-West University, Centre of Excellence for Nutrition, Potchefstroom \\ Correspondence to: Una Maclntyre, e-mail: unam@mweb.co.za \\ Keywords: cross-sectional, micronutrient dilution, added sugar, Africans, nutrition transition
}

Abstract

Objective: The objective was to investigate the absolute micronutrient intake and the possibility of micronutrient dilution of added sugar in the diets of an African population in nutritional transition.

Design: A cross-sectional, comparative, population-based design was used. Respondents who consumed sugar were divided into four quartiles of percentage of added sugar intake.

Setting: The setting was 37 randomly selected rural and urban areas of the North West province.

Subjects: The subjects were 1742 adult volunteers ( 739 men and 1003 women), aged between 15-65 years. After exclusion of low-energy reporters, the sample comprised 1045 subjects (472 men and 573 women).

Outcome measures: The outcomes measures were the macronutrient and micronutrient intakes of subjects in different quartiles of added sugar intake and body mass index (BMI).

Results: The average intake of added sugar was $10.01 \%$ of total energy $(67.12 \mathrm{~g})$ in men and $11.2 \%$ total energy $(67.10 \mathrm{~g})$ in women. Respondents who consumed the most added sugar had significantly lower mean intakes of alcohol, but higher intakes of energy, macronutrients and most micronutrients. The diets of those in the highest sugar intake group contained significantly less thiamine, riboflavin, niacin, vitamin $\mathrm{B}_{12}$, pantothenic acid, biotin, magnesium, phosphorus and zinc per 4.18 MJ. At every level of added sugar consumption, the mean intakes of fibre (men only), folate, ascorbic acid and calcium (men and women) did not meet the dietary reference intakes [estimated average requirements (EAR)] and pantothenic acid and biotin (women only) did not meet the adequate intake. There were no significant differences in mean BMI across the quartiles of added sugar intakes in men, but the mean BMI of women who consumed the most added sugar was significantly higher than that of those who consumed less sugar. Respondents who consumed the most added sugar had significantly higher intakes of fruit (men only), bread and soft drinks, and lower intakes of maize meal and alcohol (men and women).

Conclusion: Absolute intakes of most micronutrients were significantly higher in consumers with a high sugar intake [Quartile (Q) 4] compared with the lowest consumers of sugar (Q1). The lowest percentages of participants whose micronutrient intakes fell below the EAR were in Q4 and Q3. However, expression of micronutrient intake per $4.18 \mathrm{~kJ}$ (micronutrient dilution) revealed significantly less of most micronutrients per 4.18 MJ for men and women who consumed the most added sugar, compared with those who consumed the least.

(P) Peer reviewed. (Submitted: 2011-10-28. Accepted: 2012-03-11.) ๑ SAJCN

S Afr J Clin Nutr 2012;25(3):122-130

\section{Introduction}

African people living in South Africa are rapidly changing their lifestyles by moving to more urban areas. Potentially, urban areas provide more jobs and higher incomes, resulting in improvements in living standards. A change in eating patterns is an aspect of urbanisation that can negatively affect health. Traditional diets that are high in fibre and complex carbohydrates are being substituted with foods that contain more fat and refined carbohydrates, including food and drinks that contain added sugar. ${ }^{1}$ Some forms of sugar, such as fructose in fruit and lactose in milk, occur naturally in foods and add nutrients to the diet. On the other hand, added sugar does not occur naturally, and is defined as sugar that is added to food during processing or preparation. Increased consumption of added sugars can result in a decreased intake of certain micronutrients. ${ }^{2}$ In some studies, high intakes of added sugar have been shown to cause micronutrient dilution (a decrease in micronutrient intake relative to total energy intake). ${ }^{3-5}$ Diluted micronutrient intakes, especially intakes of thiamine, riboflavin, vitamin $\mathrm{B}_{6}$, folate, vitamin $\mathrm{C}$, 
calcium, iron, magnesium, zinc and selenium were reported in elderly black South African women who consumed more than $18 \%$ of total energy as added sugar. ${ }^{5}$ Although high sugar intake worsened the dietary quality of the women, energy intake was the factor that was most predictive of micronutrient intake. ${ }^{5}$ It has been confirmed by other authors that energy intake is the major determinant of micronutrient adequacy. ${ }^{6,7}$

The evidence to support the assumption that high added sugar intakes cause micronutrient dilution is inconclusive. In a systematic review of 47 observational and intervention studies by Gibson, ${ }^{8}$ the author stated that the evidence that supported the negative impact of added sugars on micronutrient intake was usually weak, and that the recommended dietary allowances (RDA) of micronutrients could be achieved through a wide range of sugar intakes in a population with sufficient energy intake. Ruxton et al concluded that added sugar intakes that provided between $5-16 \%$ of dietary energy did not have a detrimental effect on the micronutrient intakes of adults. ${ }^{9}$

There has never been a national dietary survey on adults in South Africa. However, data from studies from 1983-2000 were analysed to determine the food items and portion sizes most consumed by South Africans. ${ }^{10}$ Added sugar was consumed by $77 \%$ of the South African adult population. There were differences in sugar intake between the urban and rural areas. ${ }^{10}$ Walker et al ${ }^{11}$ reported that adolescents living in urban areas consumed almost double the amount of sugar (118-141 g) compared to those living in rural areas (69- $97 \mathrm{~g})$. This finding was also seen in data from Nutrient Intakes of South Africans, which reported the highest intake of sugar among urban African men and women. ${ }^{12}$

Kruger et $\mathrm{al}^{13}$ reported that even though urban dwellers in the North West province who participated in the Transition and Health during Urbanisation in South Africa (THUSA) survey had higher intakes of most vitamins and iron compared with respondents living in rural areas, $50 \%$ of respondents still had intakes of iron, zinc, calcium, ascorbic acid and folate that were below $67 \%$ of the RDA. Urban subjects ate a variety of foods, whereas rural subjects, especially those living on large commercial farms, ate mainly staple foods. Because of the lack of variety in their diets and low intakes of fruit and vegetables, these respondents had inadequate intakes of most micronutrients, which increased the risk of multiple micronutrient deficiencies. ${ }^{13}$

Added sugar may not only cause micronutrient dilution, but may also add unnecessary energy to the diet, resulting in weight gain and obesity. ${ }^{14}$ Overweight and obesity rates in South Africa are alarmingly high. More than half of South African women are overweight. ${ }^{15}$ It is recommended that sugar intake in South Africa should not exceed $55 \mathrm{~g}$ per day or $6-10 \%$ of total energy intake. ${ }^{16}$

From a public health perspective, it is important to investigate added sugar intakes and their possible micronutrient dilution effects in a population in transition and at risk of nutritional deficiencies. The THUSA survey was undertaken from 1996-1998 (Vorster et al). ${ }^{17}$ The objective of this project was to gather data on the impact of urbanisation on the health and well-being of the African population at different levels of transition. In the present study, dietary intake from this population was analysed to determine the effect of sugar intake on the intake of nutrients and food groups, specifically the absolute micronutrient intakes, as well as micronutrient dilution.

\section{Method}

\section{Study design, subject selection and organisational procedures}

A statistical model was used to recruit 1742 apparently healthy volunteers aged 15 years and older from 37 randomly selected sites in rural and urban areas. Volunteers were excluded from the study if they had any diseases, used chronic medication, or had an oral temperature above $37^{\circ} \mathrm{C}$. Pregnant and lactating women were also excluded. The study was approved by the ethics committee of NorthWest University (ethics number 4M5-95), and all participants signed an informed consent form after the procedures were explained to them in their native tongue.

Demographic information and dietary intake data were taken during individual interviews by specially trained, multilingual fieldworkers in the language of the participant's choice, using questionnaires that were specifically designed or adapted and validated for this population. Dietary intakes were measured with a culturally sensitive, quantitative food frequency questionnaire (QFFQ), which was validated against a seven-day weighed food record and biomarkers, ${ }^{18}$ as well as a combination of statistical methods. ${ }^{19}$ Books with photographs of commonly eaten foods in three portion sizes were used to establish the portion sizes of foods eaten. ${ }^{20}$ Reproducibility of the questionnaire was tested on a subsample of 144 respondents. ${ }^{21}$

Anthropometric measurements (height and weight) were taken by postgraduate biokinetics students and standardised by a level III anthropometrist. The participants wore only their underwear and measurements were taken in triplicate. Calibrated instruments (Precision Health Scale ${ }^{\circledR}$, A \& D Company, Japan; and Invicta Stadiometer ${ }^{\circledR}$, UK) were used. Fasting blood samples were analysed using standardised methodology to determine iron status in order to validate iron intake data.

Individuals' micronutrient intakes were expressed as total intakes. Micronutrient dilution was also investigated by analysing micronutrient intakes relative to total energy intake to correct for the higher energy intake of the groups consuming more added sugar. Individual and mean nutrient intakes were compared with the USA dietary recommended intakes (EAR and $\mathrm{Al})^{2,22,23}$ to assess the adequacy of micronutrient intakes.

\section{Adjustment for low-energy reporting}

Inconsistencies in the results of studies on associations between added sugars and micronutrient status may be due to misreporting errors. ${ }^{24}$ Bias in self-reported dietary intake, particularly due to under-reporting, is a well-documented phenomenon in dietary surveys and is highly likely to mask associations between added sugar and micronutrient intakes. ${ }^{25,26}$ However, in many studies in this area, researchers did not adjust for under-reporting, which appears to be more common in overweight adults, particularly for sweetened foods. ${ }^{25,26}$ Since it was previously reported that approximately $40 \%$ 
of respondents in the THUSA study possibly under-reported their energy intake, ${ }^{27}$ we followed the recommendation of Livingstone and Rennie, ${ }^{24}$ and the example of Gibson and Boyd, ${ }^{28}$ who excluded low-energy reporters (LERs) in their investigation of the association between added sugars and micronutrient intakes and status in the National Diet and Nutrition Survey of Young People in the UK. Therefore, records in which the energy intake:basal metabolic rate ratio was below 1.2 were identified as LERs ${ }^{29}$ and excluded from further analyses. Of the 739 men, $267(36.1 \%)$ were LERs, and of the 1003 women, $430(42.9 \%)$ were LERs. The mean added sugar intake of the LERs was about half that of the rest of the participants.

\section{Statistical analysis}

The average daily intake of each food item was calculated in grams from the weight of the portion size and the frequency of consumption. Portion sizes that were reported in household measures were converted to weights. The FoodFinder ${ }^{\circledR}$ dietary analysis programme of the Medical Research Council was used for the dietary analyses. The contributions of carbohydrates, sugar, protein and fat to total energy intakes were expressed as percentages of total energy intakes. The Statistical Package for the Social Sciences $15^{\circledR}$ (SPSS, Chicago, Illinois, USA) was used for statistical analyses. Sugar was divided into quartiles of intake for men and women separately. Descriptive

Table I: Mean daily nutrient intake, body mass index and age of men, according to quartiles of sugar intake ( $\mathrm{g} /$ day)

\begin{tabular}{|c|c|c|c|c|c|c|}
\hline Nutrient & $\begin{array}{c}\text { DRI } \\
\text { (EAR) }\end{array}$ & $\begin{array}{c}\text { Total } \\
(n=472)\end{array}$ & $\begin{array}{c}Q 1 \\
(n=118)\end{array}$ & $\begin{array}{c}Q 2 \\
(n=118)\end{array}$ & $\begin{array}{c}Q 3 \\
(n=117)\end{array}$ & $\begin{array}{c}Q 4 \\
(n=119)\end{array}$ \\
\hline Added sugar (g) & & 67.1 & $18.0^{a, b, c}$ & $43.9^{\mathrm{a}, \mathrm{d}, \mathrm{e}}$ & $70.2^{\mathrm{b}, \mathrm{e}, \mathrm{f}}$ & $136.0^{\mathrm{c}, \mathrm{d}, \mathrm{f}}$ \\
\hline$\%$ energy from sugar & & $10.01(9.4,10.6)$ & $3.06^{a, b, c}$ & $7.06^{\mathrm{a}, \mathrm{d}, \mathrm{e}}$ & $10.90^{\mathrm{b}, \mathrm{e}, \mathrm{f}}$ & $18.92^{\mathrm{c}, \mathrm{d}, \mathrm{f}}$ \\
\hline Alcohol $(\mathrm{g})$ & & 21.9 & $33.0^{\mathrm{a}}$ & $29.6^{b}$ & 24.7 & $16.6^{\mathrm{a}, \mathrm{b}}$ \\
\hline $\begin{array}{l}\text { Total energy (kJ) (including } \\
\text { alcohol) }\end{array}$ & & 11562 & $10586^{a, b}$ & $11331^{c}$ & $11500^{\mathrm{a}, \mathrm{d}}$ & $12817^{\mathrm{b}, \mathrm{c}, \mathrm{d}}$ \\
\hline Protein $(g)$ & 58 & 77.3 & $72.9^{a, b}$ & $79.2^{\mathrm{a}}$ & 77.1 & $80.0^{\mathrm{b}}$ \\
\hline$\%$ energy protein & & 11.5 & $12.1^{\mathrm{a}, \mathrm{b}}$ & $12.0^{c, d}$ & $11.4^{\mathrm{a}, \mathrm{c}, \mathrm{e}}$ & $10.4^{\mathrm{b}, \mathrm{d}, \mathrm{e}}$ \\
\hline Fat $(g)$ & & 68.6 & $58.2^{\mathrm{a}, \mathrm{b}, \mathrm{c}}$ & $68.2^{\mathrm{a}}$ & $73.2^{b}$ & $74.6^{\mathrm{c}}$ \\
\hline$\%$ energy fat & & 23.7 & $22.8^{a}$ & 24.3 & $25.2^{a, b}$ & $22.6^{b}$ \\
\hline Carbohydrate (g) & & 417.9 & $373.8^{\mathrm{a}}$ & $394.2^{b}$ & $407.0^{c}$ & $495.2^{a, b, c}$ \\
\hline$\%$ energy carbohydrates & & 67.3 & $66.8^{a}$ & $65.3^{b}$ & $65.4^{c}$ & $69.4^{\mathrm{a}, \mathrm{b}, \mathrm{c}}$ \\
\hline Fibre $(g)$ & 30 & 21.9 & $20.4^{\mathrm{a}}$ & 21.8 & 22.6 & $23.0^{\mathrm{a}}$ \\
\hline Vitamin A ( $\mu \mathrm{g} R \mathrm{R})$ & 625 & 787.2 & $632.8^{\mathrm{a}, \mathrm{b}, \mathrm{c}}$ & $885.8^{a}$ & $826.5^{b}$ & $803.5^{c}$ \\
\hline Thiamine (mg) & 1 & 1.41 & 1.38 & 1.45 & 1.39 & 1.43 \\
\hline Riboflavin (mg) & 1.1 & 1.73 & 1.68 & 1.83 & 1.72 & 1.71 \\
\hline Niacin (mg) & 12 & 18.2 & 17.6 & 19.4 & 18.1 & 17.6 \\
\hline Vitamin $B_{12}(\mu \mathrm{g})$ & 2 & 5.5 & 4.6 & 6.1 & 6.0 & 5.3 \\
\hline Vitamin $B_{6}(m g)$ & 1.1 & 1.29 & $1.13^{\mathrm{a}, \mathrm{b}, \mathrm{c}}$ & $1.36^{\mathrm{a}}$ & $1.35^{\mathrm{b}}$ & $1.33^{c}$ \\
\hline Folate $(\mu \mathrm{g})$ & 320 & 257.9 & 239.0 & 363.1 & 264.3 & 265.4 \\
\hline Pantothenic acid (mg) & $5(\mathrm{Al})$ & 4.8 & 4.6 & 4.9 & 4.9 & 5.0 \\
\hline Biotin $(\mu g)$ & 30 (Al) & 32.4 & 31.7 & 32.5 & 32.0 & 33.4 \\
\hline Vitamin D $(\mu \mathrm{g})$ & 10 & 5.5 & $4.8^{\mathrm{a}}$ & 5.6 & 5.4 & $6.4^{\mathrm{a}}$ \\
\hline Vitamin E (mg) & 12 & 13.4 & $11.8^{\mathrm{a}}$ & 13.0 & 13.7 & $14.9^{\mathrm{a}}$ \\
\hline Ascorbic acid (mg) & 75 & 40.3 & $30.1^{\mathrm{a}, \mathrm{b}, \mathrm{c}}$ & $41.6^{a}$ & $44.2^{b}$ & $45.4^{c}$ \\
\hline Calcium (mg) & $800(\mathrm{Al})$ & 535.8 & $478.1^{\mathrm{a}}$ & 534.4 & 549.4 & $580.5^{a}$ \\
\hline Iron (mg) & 6 & 10.8 & $9.3^{\mathrm{a}, \mathrm{b}, \mathrm{c}}$ & $11.4^{\mathrm{a}}$ & $10.9^{b}$ & $11.6^{c}$ \\
\hline Magnesium (mg) & 350 & 432.3 & 438.8 & 450.9 & 416.2 & 423.1 \\
\hline Phosphorus (mg) & 700 & 1388 & 1354 & 1429 & 1374 & 1395 \\
\hline Zinc (mg) & 9.4 & 10.4 & $9.4^{\mathrm{a}, \mathrm{b}, \mathrm{c}}$ & $10.9^{a}$ & $10.6^{b}$ & $10.6^{c}$ \\
\hline Body mass index & & 20.7 & 20.6 & 21.1 & 20.5 & 20.6 \\
\hline Age (years) & & 36.6 & $40.7^{a, b}$ & $38.3^{c}$ & $33.4^{\mathrm{a}, \mathrm{c}}$ & $34.5^{b}$ \\
\hline
\end{tabular}

$a, b, c, d, e, f:$ Means with the same letter differ significantly, $p$-value $\leq 0.05$

Al: adequate intake,DRI: dietary reference intake, EAR: estimated average requirement, ${ }^{22,23} \mathrm{Q}$ : quartile, RE: retinol equivalent 
statistics were calculated for continuous variables. Significant differences were calculated using multivariate nonparametric tests. Spearman rank correlation coefficients were calculated between iron and ascorbic acid intakes and biomarkers of iron status.

\section{Results}

The mean intake of added sugar as a percentage of total energy for the plausible reporters was $10.5 \%$. The mean added sugar intake was $67.1 \mathrm{~g}$ (10\% of total energy [confidence interval (Cl) $9.4-10.6 \%$ ] for the men and $67.1 \mathrm{~g}(11.2 \%$ of total energy, $\mathrm{Cl} 10.2-11.8 \%)$ for the women. The mean added sugar intake of the LER men and women were $31.1 \mathrm{~g}$ and $32.8 \mathrm{~g}$ respectively, which differed significantly

Table II: Mean daily nutrient intake, body mass index and age of women, according to quartiles of sugar intake ( $\mathrm{g} /$ day)

\begin{tabular}{|c|c|c|c|c|c|c|}
\hline Nut & $\begin{array}{l}\text { DRls } \\
\text { (EARs) }\end{array}$ & $\begin{array}{c}\text { Total } \\
(n=573)\end{array}$ & $\begin{array}{c}Q 1 \\
(n=143)\end{array}$ & $\begin{array}{c}Q 2 \\
(n=143)\end{array}$ & $\begin{array}{c}03 \\
(n=142)\end{array}$ & $\begin{array}{c}Q 4 \\
(n=145)\end{array}$ \\
\hline Added sugar (g) & & 67.1 & $19.4^{a, b, c}$ & $42.0^{\mathrm{a}, \mathrm{d}, \mathrm{e}}$ & $67.2^{b, d, f}$ & $139.2^{\mathrm{c}, \mathrm{e}}$ \\
\hline $\begin{array}{l}\% \text { energy from } \\
\text { sugar }\end{array}$ & & 11.2 & $3.9^{a, b, c}$ & $8.0^{\mathrm{a}, \mathrm{d}, \mathrm{e}}$ & $12.1^{b, d, f}$ & $20.7^{c, e, f}$ \\
\hline Alcohol (g) & & 4.0 & $7.4^{\mathrm{a}, \mathrm{b}}$ & 4.2 & $2.9^{\mathrm{a}}$ & $1.8^{\mathrm{b}}$ \\
\hline $\begin{array}{l}\text { Total energy (kJ) } \\
\text { (including alcohol) }\end{array}$ & & 9901 & $8723^{a, b, c}$ & $9338^{\mathrm{a}, \mathrm{d}, \mathrm{e}}$ & $9898^{b, d, f}$ & 11633 \\
\hline Protein (g) & 46 & 77.3 & $65.6^{\mathrm{a}}$ & 69.8 & $68.9^{b}$ & $73.8^{\mathrm{a}, \mathrm{b}}$ \\
\hline$\%$ energy protein & & 11.4 & $12.3^{a, b}$ & $12.1^{\mathrm{c}, \mathrm{d}}$ & $11.2^{\mathrm{a}, \mathrm{c}, \mathrm{e}}$ & $10.1^{\mathrm{b}, \mathrm{d}, \mathrm{e}}$ \\
\hline Fat $(\mathrm{g})$ & & 65.7 & $55.2^{\mathrm{a}, \mathrm{b}, \mathrm{c}}$ & $67.0^{\mathrm{a}, \mathrm{d}}$ & $66.5^{\mathrm{b}, \mathrm{e}}$ & $74.0^{\mathrm{c}, \mathrm{d}, \mathrm{e}}$ \\
\hline$\%$ energy fat & & 25.0 & $24.2^{\mathrm{a}}$ & $27.2^{a, b, c}$ & $25.1^{b}$ & $23.5^{c}$ \\
\hline Carbohydrate (g) & & 371.4 & $322.6^{a, b}$ & $332.6^{c, d}$ & $371.4^{\mathrm{a}, \mathrm{c}, \mathrm{e}}$ & $458.5^{\mathrm{b}, \mathrm{c}}$ \\
\hline $\begin{array}{l}\text { \% energy } \\
\text { carbohydrates }\end{array}$ & & 65.4 & $65.2^{\mathrm{a}, \mathrm{b}}$ & $62.3^{\mathrm{c}, \mathrm{d}}$ & $65.5^{c, e}$ & $68.7^{\mathrm{b}, \mathrm{d}, \mathrm{e}}$ \\
\hline Fibre $(\mathrm{g})$ & 21 & 20.3 & $18.9^{a, b}$ & $19.6^{c, d}$ & $21.3^{\mathrm{a}, \mathrm{c}}$ & $21.6^{\mathrm{b}, \mathrm{d}}$ \\
\hline Vitamin $A(\mu \mathrm{g} R E)$ & 500 & 881.3 & $697.6^{a, b, c}$ & $919.2^{\mathrm{a}}$ & $926.0^{\mathrm{b}}$ & $981.5^{c}$ \\
\hline Thiamine (mg) & 0.9 & 1.3 & 1.3 & 1.3 & 1.4 & 1.4 \\
\hline Riboflavin (mg) & 0.9 & 1.6 & $1.5^{\mathrm{a}}$ & 1.6 & $1.5^{\mathrm{b}}$ & $1.7^{a b}$ \\
\hline Niacin (mg) & 11 & 15.2 & $14.0^{\mathrm{ab}}$ & 15.3 & $15.7^{\mathrm{a}}$ & $15.7^{b}$ \\
\hline Vitamin $B_{12}(\mu \mathrm{g})$ & 2 & 5.4 & 4.7 & 5.8 & 5.4 & 5.9 \\
\hline Vitamin $B_{6}(\mathrm{mg})$ & 1.1 & 1.2 & $1.0^{\mathrm{a}, \mathrm{b}, \mathrm{c}}$ & $1.2^{\mathrm{a}}$ & $1.3^{\mathrm{b}}$ & $1.3^{\mathrm{c}}$ \\
\hline Folate $(\mu \mathrm{g})$ & 320 & 224 & $207^{a, b}$ & 225 & $228^{a}$ & $237^{b}$ \\
\hline $\begin{array}{l}\text { Pantothenic acid } \\
\text { (mg) }\end{array}$ & 5.0 (Al) & 4.0 & $3.8^{\mathrm{a}, \mathrm{b}}$ & $4.1^{\mathrm{a}}$ & $4.0^{c}$ & $4.4^{b, c}$ \\
\hline Biotin $(\mu \mathrm{g})$ & 30.0 (Al) & 24.9 & 24.3 & 24.7 & $23.5^{\mathrm{a}}$ & $27.1^{\mathrm{a}}$ \\
\hline Vitamin D $(\mu \mathrm{g})$ & 5 & 4.6 & 4.4 & 4.6 & 4.3 & 5.2 \\
\hline Vitamin E (mg) & 12 & 12.2 & $10.3^{a, b, c}$ & $12.5^{\mathrm{a}}$ & $12.3^{b}$ & $13.7^{c}$ \\
\hline Ascorbic acid (mg) & 60 & 45.7 & $37.1^{\mathrm{a}, \mathrm{b}}$ & $48.9^{\mathrm{a}}$ & $48.9^{b}$ & 47.6 \\
\hline Calcium (mg) & 800 (Al) & 489.9 & $452.6^{a}$ & $471.7^{b}$ & 494.0 & $541.2^{\mathrm{a}, \mathrm{b}}$ \\
\hline Iron (mg) & 8.1 & 10.5 & $9.4^{\mathrm{a}, \mathrm{b}}$ & $10.2^{c}$ & $11.1^{\mathrm{a}}$ & $11.4^{b, c}$ \\
\hline Magnesium (mg) & 265 & 359.4 & 353.1 & 348.7 & 366.6 & 369.2 \\
\hline $\begin{array}{l}\text { Phosphorus (mg) } \\
\text { Zinc (mg) }\end{array}$ & $\begin{array}{l}700 \\
6.8\end{array}$ & $\begin{array}{c}1166 \\
9.6\end{array}$ & $\begin{array}{l}2160^{\mathrm{a}} \\
8.9^{\mathrm{a}, \mathrm{b}, \mathrm{c}}\end{array}$ & $\begin{array}{c}2177 \\
9.7^{\mathrm{a}}\end{array}$ & $\begin{array}{l}2210 \\
9.8^{b}\end{array}$ & $\begin{array}{c}2259^{a} \\
10.1^{c}\end{array}$ \\
\hline Body mass index & & 25.8 & $25.0^{\mathrm{a}}$ & 25.8 & $25.3^{b}$ & $26.9^{\mathrm{ab}}$ \\
\hline Age (years) & & 36.2 & 37.7 & 35.2 & 36.4 & 35.7 \\
\hline
\end{tabular}

a, b, c, d, e, f: Means with the same letter differ significantly, p-value $\leq 0.05$

Al: adequate intake, DRI: dietary reference intake, ${ }^{22,23}$ EAR: estimated average requirement, Q: quartile
( $p$-value $<0.001)$ from the plausible energy reporters. The mean intakes of added sugar were 18.0, 43.9, 70.2 and $136.0 \mathrm{~g}$ for the men, and 19.4, 42.0, 67.2 and $139.2 \mathrm{~g}$ for the women in the four quartiles, respectively. On average, women consumed more sugar than men did. Male farm workers consumed significantly more sugar than upper-class urban men, who consumed the least added sugar. There were no significant differences in the mean added sugar intake between the women in the different strata of urbanisation (data not shown here). ${ }^{27}$

Mean intakes of energy, macronutrients and micronutrients, and mean BMI and age of men and women across quartiles are shown in Tables I and II.

Total energy, protein, fat and carbohydrate intakes were significantly higher in Q4 compared with Q1 for both men and women.

Alcohol intakes decreased with higher added sugar intake. Dietary fibre intake was low for all the groups. BMI did not differ among male respondents with the highest and lowest added sugar intake, but the mean BMls of women in Q4 were significantly higher than those in Q1 and Q3. Comparison of the absolute intake of micronutrients by men in the different quartiles of sugar intake revealed that those who consumed the most sugar (and energy, Q4) also consumed significantly more vitamin $\mathrm{A}$, vitamin $\mathrm{B}_{6}$, vitamin $\mathrm{D}, \mathrm{E}$ and $\mathrm{C}$, calcium, iron and zinc. Women in $Q 4$ also consumed significantly more vitamin $A$, riboflavin, niacin, vitamin $B_{6}$, folate, pantothenic acid, calcium, iron, phosphorus and zinc than those in Q1.

The mean intakes of fibre (men only), folate, ascorbic acid and calcium (men and women) did not meet the DRI (EAR). ${ }^{22,23}$ The mean intakes of pantothenic acid and biotin (women only) did not meet the Al at any level of added sugar intake. The mean intakes of most of the other micronutrients met the daily reference intakes (DRIs) at all levels of added sugar consumption for both men and women. However, in Q1, the DRls for vitamins $E$ (men and women) and vitamin $D$ (men) and $B_{6}$ (women) were not met. When the percentage of participants whose micronutrient intakes fell below the EAR was compared among the quartiles of added sugar intake, the lowest percentage of men and women with intakes below the EAR was in Q4 (not shown here) followed by the percentage in Q3. No statistically significant correlations were found between intakes of iron and ascorbic acid and any of the biomarkers of iron status (data not shown here).

The micronutrient density of the diets is shown in Tables III and IV.

A negative trend was observed when micronutrient intake was expressed per 4.18 MJ. The mean intakes of thiamine, riboflavin, niacin, pantothenic acid, biotin, magnesium, phosphorus and zinc per 4.18 MJ were significantly less for the men consuming the most added sugar (Q4) compared with those consuming the least added sugar (Q1) ( $p$-value $=0.05)$. For women consuming the most added sugar (Q4), the mean intakes of thiamine, riboflavin, niacin, vitamin $B_{12}$, folate, pantothenic acid, biotin, calcium, magnesium, phosphorus, zinc and iron per 4.18 MJ were also significantly less than women consuming the least added sugar (in Q1) ( $p$-value $<0.05)$. 
Table III: Mean daily micronutrient intakes of men (per 4.18 MJ), according to quartiles of added sugar intake as a proportion of energy (\% energy)

\begin{tabular}{|c|c|c|c|c|}
\hline Micronutrient & $\begin{array}{c}Q 1 \\
(n=118)\end{array}$ & $\begin{array}{c}Q 2 \\
(n=118)\end{array}$ & $\begin{array}{c}Q 3 \\
(n=117)\end{array}$ & $\begin{array}{c}Q 4 \\
(n=119)\end{array}$ \\
\hline Added sugar (g) & $18.0^{\mathrm{a}, \mathrm{b}, \mathrm{c}}$ & $43.9^{\mathrm{a}, \mathrm{d}, \mathrm{e}}$ & $70.2^{\mathrm{b}, \mathrm{e}, \mathrm{f}}$ & $136.0^{\mathrm{c}, \mathrm{d}, \mathrm{f}}$ \\
\hline$\%$ energy from sugar & $3.06^{a, b, c}$ & $7.06^{\mathrm{a}, \mathrm{d}, \mathrm{e}}$ & $10.90^{b, e, f}$ & $18.92^{\mathrm{c}, \mathrm{d}, \mathrm{f}}$ \\
\hline Vitamin A ( $\mu \mathrm{g} / 4.18 \mathrm{MJ})$ & $260.3^{\mathrm{a}}$ & $327.9^{\mathrm{a}}$ & 316.3 & 272.8 \\
\hline Thiamine (mg/4.18 MJ) & $0.55^{a, b}$ & $0.54^{c}$ & $0.51^{\mathrm{a}, \mathrm{d}}$ & $0.47^{b, c, d}$ \\
\hline Ribolavin (mg/4.18 MJ) & $0.67^{\mathrm{a}}$ & $0.60^{\mathrm{b}}$ & $0.63^{c}$ & $0.57^{\mathrm{a}, \mathrm{b}, \mathrm{c}}$ \\
\hline Niacin (mg/4.18 MJ) & $6.8^{\mathrm{a}}$ & $7.1^{\mathrm{b}, \mathrm{c}}$ & $6.5^{\mathrm{b}, \mathrm{d}}$ & $5.8^{\mathrm{a}, \mathrm{c}, \mathrm{d}}$ \\
\hline Vitamin $B_{12}(\mu \mathrm{g} / 4.18 \mathrm{MJ})$ & $0.45^{\mathrm{a}}$ & $0.51^{a, b}$ & 0.49 & $0.45^{b}$ \\
\hline Vitamin $B_{6}(\mathrm{mg} / 4.18 \mathrm{MJ})$ & 1.9 & 2.2 & 2.3 & 1.8 \\
\hline Folate $(\mu \mathrm{g} / 4.18 \mathrm{MJ})$ & $12.5^{\mathrm{a}}$ & 15.6 & $16.7^{\mathrm{a}}$ & 15.5 \\
\hline Pantothenic acid (mg/4.18 MJ) & $1.86^{\mathrm{a}}$ & $1.83^{b}$ & $1.80^{c}$ & $1.67^{\mathrm{a}, \mathrm{b}, \mathrm{c}}$ \\
\hline Biotin $(\mu \mathrm{g} / 4.18 \mathrm{MJ})$ & $12.9^{\mathrm{a}, \mathrm{b}}$ & 12.1 & $11.5^{\mathrm{a}}$ & $11.1^{\mathrm{b}}$ \\
\hline Vitamin D ( $\mu \mathrm{g} / 4.18 \mathrm{MJ})$ & 1.8 & 2.4 & 3.6 & 2.3 \\
\hline Vitamin E (mg/4.18 MJ) & 4.8 & 5.6 & 5.5 & 5.6 \\
\hline Ascorbic acid (mg/4.18 MJ) & 13.4 & 16.6 & 17.6 & 16.1 \\
\hline Calcium (mg/4.18 MJ) & 196.0 & 201.0 & 202.4 & 192.5 \\
\hline Iron (mg/4.18 MJ) & $3.7^{\mathrm{a}}$ & $4.3^{\mathrm{a}, \mathrm{b}}$ & 4.0 & $3.8^{b}$ \\
\hline Magnesium (mg/4.18 MJ) & $169.3^{\mathrm{a}, \mathrm{b}}$ & $163.6^{c, d}$ & $150.1^{\mathrm{a}, \mathrm{c}, \mathrm{e}}$ & $138.5^{\mathrm{b}, \mathrm{d}, \mathrm{e}}$ \\
\hline Phosphorus (mg/4.18 MJ) & $532.7^{\mathrm{a}, \mathrm{b}}$ & $524.7^{c, d}$ & $498.6^{\mathrm{a}, \mathrm{c}, \mathrm{e}}$ & $457.9^{\mathrm{b}, \mathrm{d}, \mathrm{e}}$ \\
\hline Zinc (mg/4.18 MJ) & 3.8 & $4.0^{\mathrm{a}}$ & $3.9^{b}$ & $3.5^{\mathrm{a}, \mathrm{b}}$ \\
\hline
\end{tabular}

Table IV: Mean daily micronutrient intakes of women (per $4.18 \mathrm{MJ}$ ), according to quartiles of sugar intake as a proportion of energy (\% energy)

\begin{tabular}{|c|c|c|c|c|}
\hline Micronutrient & $\begin{array}{c}Q 1 \\
(n=143)\end{array}$ & $\begin{array}{c}Q 2 \\
(n=143)\end{array}$ & $\begin{array}{c}03 \\
(n=142)\end{array}$ & $\begin{array}{c}Q 4 \\
(n=145)\end{array}$ \\
\hline Added sugar $(\mathrm{g})$ & $19.4^{\mathrm{a}, \mathrm{b}, \mathrm{c}}$ & $42^{\mathrm{a}, \mathrm{d}, \mathrm{e}}$ & $67.2^{\mathrm{b}, \mathrm{d}, \mathrm{f}}$ & $139.2^{c, e, f}$ \\
\hline$\%$ energy from sugar & $3.9^{a, b, c}$ & $8^{\mathrm{a}, \mathrm{d}, \mathrm{e}}$ & $12.1^{b, d, f}$ & $20.7^{\mathrm{ce}, \mathrm{e}, \mathrm{f}}$ \\
\hline Vitamin A ( $\mu \mathrm{g} / 4.18 \mathrm{MJ})$ & 342.8 & 415.4 & 394.6 & 361.4 \\
\hline Thiamine (mg/4.18 MJ) & $0.62^{\mathrm{a}, \mathrm{b}, \mathrm{c}}$ & $0.58^{\mathrm{a}, \mathrm{d}}$ & $0.57^{\mathrm{b}, \mathrm{e}}$ & $0.49^{c, d, e}$ \\
\hline Riboflavin (mg/4.18 MJ) & $0.72^{a, b}$ & $0.74^{\mathrm{c}, \mathrm{d}}$ & $0.66^{a, c}$ & $0.62^{b, d}$ \\
\hline Niacin (mg/4.18 MJ) & $6.7^{\mathrm{a}}$ & $6.8^{b}$ & $6.6^{c}$ & $5.6^{\mathrm{a}, \mathrm{b}, \mathrm{c}}$ \\
\hline Vitamin $B_{12}(\mu \mathrm{g} / 4.18 \mathrm{MJ})$ & $0.5^{\mathrm{a}, \mathrm{b}}$ & $0.55^{\mathrm{a}, \mathrm{c}}$ & $0.54^{b, d}$ & $0.47^{c, d}$ \\
\hline Vitamin $\mathrm{B}_{6}(\mathrm{mg} / 4.18 \mathrm{MJ})$ & 2.3 & 2.6 & 2.4 & 2.1 \\
\hline Folate $(\mu \mathrm{g} / 4.18 \mathrm{MJ})$ & 18 & $22.1^{\mathrm{a}}$ & $22.3^{b}$ & $17.4^{a, b}$ \\
\hline Pantothenic acid (mg/4.18 MJ) & $1.9^{\mathrm{a}, \mathrm{b}}$ & $1.9^{c, d}$ & $1.7^{\mathrm{a}, \mathrm{c}, \mathrm{e}}$ & $1.6^{\mathrm{b}, \mathrm{d}, \mathrm{e}}$ \\
\hline Biotin $(\mu \mathrm{g} / 4.18 \mathrm{MJ})$ & $11.9^{a, b}$ & $11.1^{\mathrm{c}, \mathrm{d}}$ & $10^{\mathrm{a}, \mathrm{c}}$ & $9.6^{b, d}$ \\
\hline Vitamin D ( $\mu \mathrm{g} / 4.18 \mathrm{MJ})$ & $2.8^{\mathrm{a}}$ & 2.3 & $1.7^{\mathrm{a}}$ & 2.2 \\
\hline Vitamin E (mg/4.18 MJ) & 5.6 & $5.9^{\mathrm{a}}$ & $5^{\mathrm{a}}$ & 5.4 \\
\hline Ascorbic acid (mg/4.18 MJ) & 18.4 & 22.4 & 22.6 & 17.5 \\
\hline Calcium (mg/4.18 MJ) & $221.3^{\mathrm{a}}$ & 216.4 & 212.6 & $195^{\mathrm{a}}$ \\
\hline Iron (mg/4.18 MJ) & $4.5^{\mathrm{a}}$ & $4.6^{\mathrm{b}}$ & $4.7^{\mathrm{c}}$ & $4.1^{a, b, c}$ \\
\hline Magnesium (mg/4.18 MJ) & $168.4^{a, b, c}$ & $155.8^{\mathrm{a}, \mathrm{d}}$ & $153.4^{\mathrm{b}, \mathrm{e}}$ & $133.7^{\mathrm{c}, \mathrm{d}, \mathrm{e}}$ \\
\hline Phosphorus (mg/4.18 MJ) & $540^{a, b, c}$ & $513.7^{\mathrm{a}, \mathrm{d}, \mathrm{e}}$ & $491.5^{b, d, f}$ & $442.3^{c, e, f}$ \\
\hline Zinc (mg/4.18 MJ) & $4.3^{\mathrm{a}}$ & $4.4^{\mathrm{b}, \mathrm{c}}$ & $4.1^{\mathrm{b}, \mathrm{d}}$ & $3.7^{\mathrm{a}, \mathrm{c}, \mathrm{d}}$ \\
\hline
\end{tabular}

$\mathrm{a}, \mathrm{b}, \mathrm{c}, \mathrm{d}, \mathrm{e}, \mathrm{f}$ : Means with the same letter differ significantly ( $\mathrm{p}$-value $\leq 0.05$ ) Q: quartile
Table V: Mean dietary intake of men according to quartiles of added sugar intake ( $\mathrm{g} /$ day, $\mathrm{n}=472)$

\begin{tabular}{|c|c|c|c|c|}
\hline Food group & $\begin{array}{c}01 \\
(n=118)\end{array}$ & $\begin{array}{c}Q 2 \\
(n=118)\end{array}$ & $\begin{array}{c}03 \\
(n=117)\end{array}$ & $\begin{array}{c}04 \\
(n=119)\end{array}$ \\
\hline Added sugar (g) & $18.0^{\mathrm{a}, \mathrm{b}, \mathrm{c}}$ & $43.9^{\mathrm{a}, \mathrm{d}, \mathrm{e}}$ & $70.2^{\mathrm{b}, \mathrm{e}, \mathrm{f}}$ & $136.0^{c, d, f}$ \\
\hline$\%$ energy from sugar & $3.06^{a, b, c}$ & $7.06^{\mathrm{a}, \mathrm{d}, \mathrm{e}}$ & $10.90^{\mathrm{b}, \mathrm{e}, \mathrm{f}}$ & $18.92^{c, d, f}$ \\
\hline Milk & 202.8 & 175.3 & 194.1 & 177.1 \\
\hline Red meat & 41.6 & 50.4 & 52.6 & 45.3 \\
\hline Chicken & 15.2 & 20.8 & 20.9 & 22.5 \\
\hline Fish & 11.3 & 16.7 & 11.9 & 11.5 \\
\hline Legumes & 40.1 & 42.6 & 47.3 & 41.5 \\
\hline Eggs & 54.1 & 58.0 & 54.1 & 64.9 \\
\hline Fruit & $88.9^{a, b}$ & 123.6 & $123.7^{\mathrm{a}}$ & $126.7^{b}$ \\
\hline Vegetables & $66.5^{\mathrm{a}, \mathrm{b}}$ & $75.7^{\mathrm{a}}$ & $82.1^{\mathrm{b}}$ & 80.1 \\
\hline Potatoes & 38.8 & 40.2 & 45 & 42.6 \\
\hline Dry mealie meal & $225.8^{\mathrm{a}, \mathrm{b}, \mathrm{c}}$ & $169.2^{b}$ & $152^{\mathrm{a}}$ & $165.4^{c}$ \\
\hline Rice & 45.9 & 55.30 & 45.2 & 47.4 \\
\hline Samp & 51.1 & 47.4 & 48.9 & 39.9 \\
\hline Bread & $99.6^{a, b}$ & 120.8 & $149.6^{a}$ & $159.4^{b}$ \\
\hline Cereal & 45.3 & 76.1 & 87.6 & 73.9 \\
\hline Fat & 10.6 & 16.6 & 12.4 & 11.3 \\
\hline Sweets & 47.2 & 44.4 & 40.0 & 46.3 \\
\hline Sugar & $9.4^{a, b, c}$ & $24.1^{\mathrm{a}}$ & $42.9^{b}$ & $77.0^{c}$ \\
\hline Soft drinks & $52.1^{\mathrm{a}}$ & $108.9^{b}$ & $148.7^{c}$ & $374.6^{\mathrm{a}, \mathrm{b}, \mathrm{c}}$ \\
\hline
\end{tabular}

$a, b, c, d$, e: Means with the same letter differ significantly ( $p$-value $\leq 0.05)$ Q: quartile

Table VI: Mean dietary intake of women according to quartiles of added sugar intake ( $\mathrm{g} /$ day, $\mathrm{n}=573$ )

\begin{tabular}{|c|c|c|c|c|}
\hline Food group & $\begin{array}{c}Q 1 \\
(n=143)\end{array}$ & $\begin{array}{c}Q 2 \\
(n=143)\end{array}$ & $\begin{array}{c}Q 3 \\
(n=142)\end{array}$ & $\begin{array}{c}04 \\
(n=145)\end{array}$ \\
\hline Added sugar (g) & $19.4^{\mathrm{a}, \mathrm{b}, \mathrm{c}}$ & $42.0^{\mathrm{a}, \mathrm{d}, \mathrm{e}}$ & $67.2^{\mathrm{b}, \mathrm{d}, \mathrm{f}}$ & $139.2^{c, e, f}$ \\
\hline$\%$ energy from sugar & $3.9^{\mathrm{a}, \mathrm{b}, \mathrm{c}}$ & $8.0^{\mathrm{a}, \mathrm{d}, \mathrm{e}}$ & $12.1^{\mathrm{b}, \mathrm{d}, \mathrm{f}}$ & $20.7^{c, e, f}$ \\
\hline Milk & 196.3 & 184.0 & 173.0 & 183.9 \\
\hline Red meat & 41.3 & 56.5 & 48.3 & 49.2 \\
\hline Chicken & 15.2 & 20.8 & 20.9 & 22.5 \\
\hline Fish & 14.1 & 14.1 & 11.6 & 11.7 \\
\hline Legumes & 46.4 & 47.2 & 43.9 & 38.2 \\
\hline Eggs & 52.3 & 45.0 & 38.8 & 46.6 \\
\hline Fruit & $90.4^{a}$ & 130.0 & $159.4^{\mathrm{a}}$ & 141.4 \\
\hline Vegetables & $71.5^{\mathrm{a}}$ & 97.1 & $93.5^{\mathrm{a}}$ & 84.0 \\
\hline Potatoes & 35.0 & 38.1 & 41.7 & 45.5 \\
\hline Mealie meal (dry) & $186.3^{a, b, c}$ & $144.6^{a}$ & $128.3^{b}$ & $144.7^{c}$ \\
\hline Rice & 38.3 & 45.6 & 43.6 & 48.8 \\
\hline Samp & 46.7 & 50.4 & 40.4 & 37.3 \\
\hline Bread & $89.7^{a, b}$ & 111.1 & $132.3^{\mathrm{a}}$ & $134.3^{b}$ \\
\hline Cereal & 68.2 & 78.7 & 101.8 & 92.8 \\
\hline Fat & 14.1 & 14.4 & 11.0 & 11.4 \\
\hline Sweets & 59.9 & 39.7 & 52.4 & 49.2 \\
\hline Sugar & $10.1^{\mathrm{a}, \mathrm{b}, \mathrm{c}}$ & $24.4^{\mathrm{a}, \mathrm{d}, \mathrm{e}}$ & $40.2^{\mathrm{b}, \mathrm{d}, \mathrm{f}}$ & $79.8^{c, e, f}$ \\
\hline Soft drinks & $55.8^{\mathrm{a}}$ & $104.3^{b}$ & $137.9^{c}$ & $382.4^{a, b, c}$ \\
\hline
\end{tabular}

$a, b, c, d, e, f:$ Means with the same letter differ significantly $(p-v a l u e ~ \leq 0.05)$ $\mathrm{Q}$ : quartile 
To understand how the intake of micronutrients differed across quartiles of added sugar intake, food consumption for different levels of added sugar intake were compared (Tables V and VI). Men consuming the most sugar had significantly higher intakes of the fruit, bread and soft drink groups and lower intakes of maize meal, whereas women consuming the most added sugar had higher intakes of the bread and soft drink groups and lower intakes of maize meal ( $p$-value $<0.05)$.

Biochemical measures of nutrient status provide a useful means of validating dietary findings. Mean values of serum ferritin, iron, ironbinding capacity, transferrin saturation and haemoglobin fell in the normal range for adults and did not differ significantly between the quartiles of added sugar for men or women (not shown here).

\section{Discussion}

To the best of our knowledge, this study is the first to report on the effect of sugar intake on micronutrient intake and dilution in South African adults (excluding the elderly). Total energy and absolute intakes of macronutrients and most micronutrients were significantly higher in consumers with a high sugar intake (Q4) compared with the lowest consumers of sugar (Q1). When the percentage of participants whose micronutrient intakes fell below the EAR was compared among the quartiles of added sugar intake, the lowest percentages of men and women with intakes below the EAR were in Q3 and Q4. This finding is in line with the higher energy intakes in Q3 and Q4 compared to Q1 and Q2. However, expression of micronutrient intake per $4.18 \mathrm{~kJ}$ (micronutrient dilution) revealed significantly less of all the micronutrients tested for men and women who consumed the most added sugar, compared with those who consumed the least added sugar.

Some studies have shown that high sugar intakes may result in micronutrient dilution. ${ }^{3-5}$ However, from the systematic reviews of Gibson ${ }^{8}$ and Rennie and Livingstone ${ }^{30}$ on the results of both observational and intervention studies, it appeared that the results were not necessarily linear or consistent across nutrients, populations and age groups; that quantification was hampered by differences in cut-offs to define categories of added sugar intake, ways of expressing sugar intake and methods of analyses.

The present study reported higher mean energy intakes for men and women than those reported in other South African surveys of rural ${ }^{31}$ and urban areas. ${ }^{32}$ There was under-reporting by the respondents on the QFFQ ${ }^{26}$ which was the reason why LERs were omitted from this data set. However, there was no evidence of a difference in proportional bias or under-reporting among gender and strata in the THUSA study. ${ }^{27}$ As pointed out in the introduction, added sugar may not only cause micronutrient dilution, but may also add unnecessary energy to the diet, resulting in weight gain and obesity. ${ }^{14}$ This seems to have been the case for the women in this study as the mean BMI of women in Q4 was significantly higher than that of those in Q1 and Q3. Generally speaking, overweight and obesity are alarmingly high in South Africa. More than half of South African women are overweight. ${ }^{15}$
Despite the higher energy intakes, micronutrient intakes were not optimal in this population, as reflected by deficient intakes of folate, ascorbic acid and calcium across all quartiles of added sugar intake. These results are consistent with findings from other South African studies. ${ }^{12}$ However, the results of a theoretical modelling of the impact of fortification on micronutrient intakes indicated that some alleviation from micronutrient deficiencies was possible after it became compulsory to fortify South African staple foods in October $2003 .{ }^{33}$ This fortification might improve the mean intakes of vitamin $B_{6}$, thiamine, riboflavin, niacin and folate. However, fortification cannot solve all micronutrient deficiencies, and deficiencies of calcium and iron are still common in adults and children in South Africa. ${ }^{34}$ The present study was conducted before the mandatory fortification of staple foods in 2003. The Institute of Medicine of the National Academies recently revised the DRIs for calcium. It stated that on average, $800 \mathrm{mg}$ of calcium is appropriate for women aged 19-50 and men up to 72 years. ${ }^{23}$ Despite the lower DRI, over $85 \%$ of the participants in this study were still at risk of inadequate calcium intakes.

Added sugar contributed a mean of $10 \%$ total energy $(67.1 \mathrm{~g})$ for the men. This is in line with the World Health Organization/Food and Agricultural Organization of the United Nations recommendation of $<10 \%$ of total energy consumed, ${ }^{34}$ but is somewhat higher than the South African recommended intake of $<55 \mathrm{~g} /$ day to prevent dental caries. ${ }^{16}$ By contrast, the mean intake of the women was $11.2 \%$ total energy $(67.1 \mathrm{~g})$, which is more than the recommended $10 \%$. When setting the 2002 DRls, the American Institute of Medicine concluded that there was insufficient evidence to set an upper-intake level for added sugars. However, it suggested a maximum intake level of $25 \%$ of total energy because of "growing concerns about inadequate micronutrient intakes". 2

We used two approaches to assess the effect of added sugar on micronutrient intakes. The first approach expressed micronutrient intakes as absolute daily intake. The results of this approach showed that participants who consumed more added sugar had higher absolute intakes of most micronutrients. These results concur with other studies that have investigated absolute intakes. ${ }^{3-5}$ However, reported total energy intake is an important indicator of micronutrient intake, and in general, is significantly positively associated with micronutrient intakes. ${ }^{24}$ Using the second approach to determine the dilution effect of added sugar (micronutrient intake per $4.18 \mathrm{MJ}$ ), a slight but significant nutrient dilution was found for thiamine, riboflavin, niacin, vitamin $\mathrm{B}_{6}$, magnesium, phosphorus and zinc in the men who consumed the most added sugar. The mean intake per $4.18 \mathrm{MJ}$ of riboflavin, niacin, magnesium and phosphorus was also slightly, but significantly, less in the women who consumed the most sugar than in the women in Q1, who consumed the least sugar. These results correlate well with another South African study ${ }^{5}$ that reported that women with the highest intakes of added sugar had lower intakes of various vitamins and minerals when expressed in relation to total energy intake.

The impact of added sugars has been shown to be very small in the literature, and it would require very large changes in sugar consumption to have any positive or negative effect on the quality 
of the diet. ${ }^{35}$ However, Overby et al ${ }^{36}$ reported a $30-40 \%$ reduction in fruit and vegetable consumption in Norwegian children and adolescents who consumed the most sugar ( $>25 \%$ of total energy).

In the current study, with regard to food intake, men who consumed the most added sugar also consumed significantly more fruit, bread and soft drinks, and women who consumed the most added sugar also consumed significantly more bread and soft drinks. Recently, the association between consumption of sugar-sweetened beverages and the risk of noncommunicable diet-related diseases has been observed by various investigators. ${ }^{37-42}$

Fortification may mask micronutrient dilution. Alexy et $\mathrm{al}^{42}$ investigated the consumption of fortified foods by German children and adolescents in the Dortmund Nutritional and Anthropometrical Longitudinally Designed (DONALD) study. They reported that $32 \%$ of food items were fortified, including beverages, sweets and cereals, and that $56 \%$ of all fortified food items in Germany contained added sugar. Similar data for South Africa are probably not available, but it is doubtful if the THUSA population had access to a similar amount of sweetened, fortified food products in 1998. Therefore, the form in which sugars are consumed appears to be an important modifier of the impact of dilution. Soft drinks, sugar and sweets are more likely to have a negative impact on diet quality, whereas dairy foods, milk drinks and pre-sweetened fortified breakfast cereals may have a positive impact. $^{?}$
The authors of a systematic review concluded that the results of 15 observational studies on added sugar intakes and micronutrient density were inconclusive and that currently, there are insufficient data to draw firm conclusions. ${ }^{24}$ Overall, the studies suggest that micronutrient intakes are optimal with moderate levels of sugar in the diet provided that energy needs are met. Very high intakes of added sugars in excess of $20 \%$ of energy are associated with lower intakes of several micronutrients. ${ }^{24} \mathrm{It}$ has been argued that low energy intakes are a better indicator of micronutrient inadequacy than dietary sugar concentration. ${ }^{7,8}$

Bias in self-reported dietary intake, particularly due to underreporting, is a well-documented phenomenon in dietary surveys ${ }^{25}$ and is highly likely to mask associations between added sugar and micronutrient intakes. However, in many studies in this area, researchers do not adjust for under-reporting, which appears to be more common in overweight adults, particularly for sweetened foods.$^{30}$ Although progress has been made in identifying misreporting in dietary surveys, consensus is required on how to adjust the data to allow for random and systematic errors in investigating the association between sugar intakes and micronutrient status.

In a recent review on the topic, Livingstone and Rennie ${ }^{26}$ discussed a range of methodological and conceptual constraints in the search for convincing evidence to either support or refute the micronutrient dilution hypothesis. They concluded that perhaps the issue could only be resolved by initiating new research that is specifically 
designed to address the issue (prospective intervention studies), with specific hypotheses and sufficient statistical power, and using dietary methodologies and statistical techniques that are fit for such purposes. They are of the opinion that "where micronutrient dilution can be attributed to added sugar, analyses should quantify the magnitude, as well as the direction, of any observed significant associations between intakes of added sugars and micronutrients".

Results of the current study, completed before it became compulsory to fortify South African staple foods in October 2003, indicate that respondents with the highest sugar intake also consumed the most food. Therefore, they also consumed the most micronutrients in terms of absolute intake. However, the micronutrient dilution effect of added sugar (micronutrient intake per 4.18 $\mathrm{MJ}$ ) was clear. A slight but significant nutrient dilution was found for thiamine, riboflavin, niacin, vitamin $B_{6}$, magnesium, phosphorus and zinc in the men who consumed the most added sugar (Q4). For women who consumed the most sugar (Q4), the mean intake of riboflavin, niacin, magnesium and phosphorus was also slightly, but significantly, less than the women in Q1, who consumed the least sugar. The respondents were black South Africans. This is an ethnic group with a high risk of inadequate micronutrient status. ${ }^{1,12}$

Further research is needed on the dietary intake of South African adults. It should represent all ethnic groups to determine nutrient deficiencies after the implementation of the fortification of staple foods and the effects of fortified sweetened products, such as breakfast cereals, on micronutrient status. It seems as though generalisations such as "sugars compromise nutrient intakes", or conversely, "micronutrient intakes are adequate irrespective of sugar intake", are unhelpful in resolving current public health issues.

\section{References}

1. Bourne LT, Lambert EV, Steyn K. Where does the black population of South Africa stand on the nutrition transition? Public Health Nutr. 2002;5(1A):157-162

2. Dietary Reference Intakes for energy, carbohydrate, fiber, fat, fatty acids, cholesterol, protein and amino acids (macronutrients). Food and Nutrition Board. Washington DC: National Academy Press, 2005; p.788.

3. Krantz S, Smiciklas-Wright H, Siega-Riz AM, Mitchell DM. Adverse effect of high added sugar consumption on dietary intake in American preschoolers. J Pediatr. 2005:146(1):105-111.

4. Charlton KE, Wolmarans P, Lombard CJ. Evidence of nutrient dilution with high sugar intakes in older South Africans. J Hum Nutr Diet. 1998;11:331-343.

5. Charlton KE, Kolbe-Alexander TL, Nel JH. Micronutrient dilution associated with added sugar intake in elderly black South African women. Eur J Clin Nutr. 2005;59(9):1030-1042.

6. Gibson SA. Dietary sugars and micronutrient dilution in normal adults aged 65 years and over Public Health Nutr. 2001;4(6):1235-1244.

7. Department of Health and Social Security: dietary sugars and human disease. Committee on Medical Aspects of Food Policy. Report on Health and Social Subjects 37. London: HMSO, 1989.

8. Gibson SA. Dietary sugars intake and micronutrient adequacy: a systematic review of the evidence. Nutr Res Rev. 2007;20:121-131.

9. Ruxton CHS, Garceau FJS, Cottrell RC. Guidelines for sugar consumption in Europe: is a quatitative approach justified? Eur J Clin Nutr. 1999;53(7):503-513.

10. Steyn NP, Nel JH, Casey A. Secondary data analyses of dietary surveys undertaken in South Africa to determine usual food consumption of the population. Public Health Nutr. 2003;6(7):631-644

11. Walker ARP, Dison E, Duvenhage A, Walker BF, et al. Dental caries in South African black and white high school pupils in relation to sugar intake and snack habits. Community Dent Oral Epidemiol. 1981;9(1):37-43.

12. Vorster HH, Oosthuizen W, Jerling J, et al. The nutritional status of South Africans: a review of the literature from 1975-1996. Durban: Health Systems Trust; 1997.

13. Kruger HS, Kruger A, Vorster HH, et al. Urbanization of Africans in the North West Province is associated with better micronutrient status: the Transition and Health during Urbanization Study in South Africa. Nutr Res. 2005;25:365-375.
14. Ludwig DS, Peterson KE, Gortmaker SL. Relation between consumption of sugarsweetened drinks and childhood obesity: a prospective observational analysis. Lancet. 2001;357(9255):505-508

15. Puoane T, Steyn K, Bradshaw D, et al. Obesity in South Africa: The South African Demographic and Health Survey. Obes Res. 2002;10(10):1038-1048.

16. Steyn NP, Myburgh NG, Nel JH. Evidence to support a food-based dietary guideline on sugar consumption in South Africa. Bulletin of the World Health Organization. 2003;81(8):599-608.

17. Vorster $\mathrm{HH}$, Venter CS, Wissing MP, Margetts BM. The nutrition and health transition in the North West Province of South Africa: a review of the THUSA (Transition and Health during Urbanization of South Africans) study. Public Health Nutr. 2005;8(5):480-490.

18. MacIntyre UE, Venter CS, Vorster HH. A culture-sensitive quantitative food frequency questionnaire used in an African population: 2. Relative validation by 7-day weighed records and biomarkers. Public Health Nutr. 2000;4(1):63-71.

19. Macintyre UE, Venter CS, Vorster HH, Steyn HS. A combination of statistical methods for the analysis of the relative validation data of the quantitative food frequency questionnaire used in the THUSA study. Public Health Nutr. 2000;4(1):45-51.

20. Venter CS, Macintyre UE, Vorster HH. The development and testing of a food portion photograph book for use in an African population. J Hum Nutr Diet. 2000;13(3):205-218.

21. Macintyre UE, Venter CS, Vorster HH. A culture-sensitive quantitative food frequency questionnaire used in an African population: 1. Development and reproducibility. Public Health Nutr. 2000;4(1):53-62.

22. Dietary Reference Intakes. Food and Nutrition Board, Institute of Medicine. Washington, DC: National Academy Press; 2003.

23. Food and Nutrition Board, Institute of Medicine for the National Academies. Dietary Reference Intakes calcium and vitamin D. In: Ross AC, Taylor CL, Yaktine AL, et al, editors. Washington, DC: National Academy Press; 2011.

24. Rennie KL, Livingstone MBE. Associations between dietary added sugar intake and micronutrient intake: a systematic review. Brit J Nutr. 2007;97(5):832-841.

25. Krebs-Smith SM, Graubard BI, Kahle LL, et al. Low energy reporters vs others: a comparison of reported food intakes. Eur J Clin Nutr. 2000;54(4):281-287.

26. Livingstone MBE, Rennie KL. Added sugars and micronutrient dilution. Obes Res. 2009;10(Suppl 1):34-40.

27. MacIntyre UE, Kruger HS, Venter CS, Vorster HH. Dietary intakes of an African population in different stages of transition in the North West Province, South Africa: the THUSA study. Nutr Res. 2002;22:239-256

28. Gibson S, Boyd A. Associations between added sugars and micronutrient intakes and status: further analysis of data from the National Diet and Nutrition Survey of Young People aged 4 to 18 years. Br J Nutr. 2009;101(1):100-107.

29. Goldberg GR, Black AE, Jebb SA, et al. Critical evaluation of energy intake data using fundamental principles of energy physiology: 1. Derivation of cut-off limits to identify underrecording. Eur J Clin Nutr. 1991;45(12):569-581.

30. Rennie KL, Livingstone MBE. Associations between dietary added sugar intake and micronutrient intake: a systematic review. Brit J Nutr. 2007;97(5):832-841.

31. Steyn NP, Burger A, Monyeki KD, et al. Variation in dietary intake of the adult population of Dikgale. S Afr J Clin Nutr. 2001;14(4):140-145

32. Bourne LT, Langenhoven ML, Steyn K, et al. Nutrient intake in the urban African population of the Cape Peninsula, South Africa: the BRISK study. Cent Afr J Med. 1993;39(12):238-247.

33. Steyn NP, Wolmarans P, Nel JH, Bourne LT. National fortification of staple foods can make a significant contribution to micronutrient intake of South African adults. Public Health Nutr. 2006;11(3):307-313.

34. World Health Organization. Diet, nutrition and the prevention of chronic diseases. Geneva: WHO; 2003.

35. Forshee RA, Storey, ML. The role of added sugars in the diet quality of children and adolescents. J Am Coll Nutr. 2001;20(1):32-43.

36. Overby NC, Lilligaard ITL, Johansson L, Andersen LF. High intake of added sugar among Norwegian children and adolescents. Public Health Nutr. 2003;7(2):285-293.

37. Johnson RK, Appel LJ, Brands M, et al. Dietary sugars intake and cardiovascular health: a scientific statement from the American HeartAssociation. Circulation. 2009;120(11):1011-1020.

38. Schulze MB, Manson JE, Ludwig DS, et al. Sugar-sweetened beverages, weight gain, and incidence of type 2 diabetes in young and middle-aged women. JAMA. 2004;292(8):927-934.

39. Malik VS, Popkin BM, Bray GA, et al. Sugar-sweetened beverages and risk of metabolic syndrome and type 2 diabetes: a meta-analysis. Diabetes Care. 2010;33(11):2477-2483.

40. De Koning L, MalikVS, Rimm EB, et al. Sugar-sweetened and artificially sweetened beverage consumption and risk of type 2 diabetes in men. Am J Clin Nutr. 2011;93(6):1321-1327.

41. Dhingra R, Sullivan L, Jacques PF, et al. Soft drink consumption and risk of developing cardiometabolic risk factors in the community. Circulation. 2007;116(5):480-488.

42. Alexy U, Sichert-Hellert W, Kersting M. Fortification masks nutrient dilution due to added sugars in the diet of children and adolescents. J Nutr. 2002;132(9):2785-2791. 